\title{
Filters and congruences in sectionally pseudocomplemented lattices and posets
}

\author{
Ivan Chajda ${ }^{1}(\mathbb{D}) \cdot$ Helmut Länger ${ }^{1,2}$ (I)
}

Accepted: 20 May 2021 / Published online: 12 June 2021

(c) The Author(s) 2021

\begin{abstract}
Together with J. Paseka we introduced so-called sectionally pseudocomplemented lattices and posets and illuminated their role in algebraic constructions. We believe that - similar to relatively pseudocomplemented lattices - these structures can serve as an algebraic semantics of certain intuitionistic logics. The aim of the present paper is to define congruences and filters in these structures, derive mutual relationships between them and describe basic properties of congruences in strongly sectionally pseudocomplemented posets. For the description of filters in both sectionally pseudocomplemented lattices and posets, we use the tools introduced by A. Ursini, i.e., ideal terms and the closedness with respect to them. It seems to be of some interest that a similar machinery can be applied also for strongly sectionally pseudocomplemented posets in spite of the fact that the corresponding ideal terms are not everywhere defined.
\end{abstract}

Keywords Sectionally pseudocomplemented lattice $\cdot$ Sectionally pseudocomplemented poset $\cdot$ Filter $\cdot$ Congruence $\cdot$ Weak regularity $\cdot$ Congruence permutability $\cdot$ Maltsev term $\cdot$ Ideal term $\cdot$ Closedness of a subset . Congruence class · Deductive system $\cdot$ Partial term

\section{Introduction}

The concept of a relative pseudocomplemented lattice was introduced by R. P.Dilworth (Dilworth (1939)). It was used in several branches of mathematics, e.g., as an algebraic axiomatization of intuitionistic logic (by Heyting and Brouwer) where the relative pseudocomplement is interpreted as the logical connective implication.

However, every relative pseudocomplemented lattice is distributive, see, e.g., Birkhoff (1979) and Lakser (1971). Because not every non-classical propositional calculus is necessarily distributive (for instance, the logic of quantum mechanics), it was a question whether the concept of relative pseudocomplementation can be extended in a rea-

Helmut Länger

helmut.laenger@tuwien.ac.at

Ivan Chajda

ivan.chajda@upol.cz

1 Department of Algebra and Geometry, Faculty of Science, Palacký University Olomouc, 17. listopadu 12, 77146 Olomouc, Czech Republic

2 Faculty of Mathematics and Geoinformation, Institute of Discrete Mathematics and Geometry, TU Wien, Wiedner Hauptstraße 8-10, 1040 Vienna, Austria sonable way to non-distributive lattices. This was realized by the first author in Chajda (2003) by introducing sectional pseudocomplementation. Later on, the concept of sectional pseudocomplementation was extended also to posets, see Chajda et al. (2021).

In the present paper we focus on congruences and filters on sectionally pseudocomplemented lattices and posets. For lattices we can use the machinery of universal algebra (see, e.g., Chajda et al. (2012)) because sectionally pseudocomplemented lattices form a variety which is congruence permutable, congruence distributive and weakly regular. The situation with sectionally pseudocomplemented posets is a bit more complicated due to the fact that such a poset in general cannot be extended to a sectionally pseudocomplemented lattice by means of the Dedekind-MacNeille completion, see Chajda et al. (2021) for the result.

Sectionally pseudocomplemented lattices having 0 and their ideals will be the topic of one of our next studies.

\section{Sectionally pseudocomplemented lattices}

Recall that a lattice $(L, \vee, \wedge)$ is said to be sectionally pseudocomplemented if for all $a, b \in L$ there exists the 
Fig. 1 Sectionally pseudocomplemented lattice that is not relatively pseudocomplemented

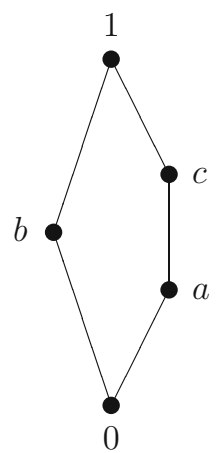

pseudocomplement of $a \vee b$ in the interval $([b), \leq)$, i.e., the greatest element $c$ of $L$ satisfying

$(a \vee b) \wedge c=b$.

(Here, $[b)$ denotes the set $\{x \in L \mid b \leq x\}$.) In this case $c$ is called the sectional pseudocomplement of a with respect to $b$ and it will be denoted by $a * b$. We consider sectionally pseudocomplemented lattices as algebras $(L, \vee, \wedge, *)$ of type $(2,2,2)$. Every non-empty sectionally pseudocomplemented lattice has a greatest element 1 , namely the algebraic constant $x * x$. In the following we consider only non-empty lattices.

An example of a sectionally pseudocomplemented lattice that is not relatively pseudocomplemented is $\mathbf{N}_{5}$ depicted in Fig. 1:

This lattice is not distributive and hence not relatively pseudocomplemented (see Birkhoff (1979)). The operation table for the sectional pseudocomplementation is as follows:

$$
\begin{array}{l|lllll}
* & 0 & a & b & c & 1 \\
\hline 0 & 1 & 1 & 1 & 1 & 1 \\
a & b & 1 & b & 1 & 1 \\
b & c & a & 1 & c & 1 \\
c & b & a & b & 1 & 1 \\
1 & 0 & a & b & c & 1
\end{array}
$$

Recall from Chajda et al. (2021), Theorems 2.5 and 2.6, the following important result.

Proposition 2.1 The class of sectionally pseudocomplemented lattices $(L, \vee, \wedge, *)$ forms a variety which besides the lattice axioms is determined by the following identities:

$$
z \vee y \leq x *((x \vee y) \wedge(z \vee y)),
$$

$(x \vee y) \wedge(x * y) \approx y$.

This variety is congruence permutable, congruence distributive and weakly regular. A Maltsev term for congruence permutability is given by

$p(x, y, z):=((x * y) * z) \wedge((z * y) * x)$.
For the concept of congruence permutability, we refer the reader to Chajda et al. (2012).

Weak regularity means that every congruence $\Theta$ on a sectionally pseudocomplemented lattice with greatest element 1 is determined by its kernel, i.e., by the congruence class $[1] \Theta$. Hence, our first task is to describe these classes. For this purpose we introduce the following concept:

Definition 2.2 Let $\mathbf{L}=(L, \vee, \wedge, *)$ be a sectionally pseudocomplemented lattice. A filter of $\mathbf{L}$ is a subset $F$ of $L$ containing 1 such that $x * y, y * x \in F$ implies

$$
\begin{aligned}
& (x \vee z) *(y \vee z),(x \wedge z) *(y \wedge z), \\
& (x * z) *(y * z),(z * x) *(z * y) \in F
\end{aligned}
$$

Let Fil $\mathbf{L}$ denote the set of all filters of $\mathbf{L}$. For any subset $M$ of $L$ define a binary relation $\Phi(M)$ on $L$ as follows:

$\Phi(M):=\left\{(x, y) \in L^{2} \mid x * y, y * x \in M\right\}$.

The following results were proved in Chajda (2003) and Chajda et al. (2021).

Lemma 2.3 If $\mathbf{L}=(L, \vee, \wedge, *)$ is a sectionally pseudocomplemented lattice and $a, b, c \in L$, then

(i) $a * b=1$ if and only if $a \leq b$,

(ii) $1 * a=a$,

(iii) $a \leq b * a$,

(iv) $a \leq(a * b) * b$,

(v) if $a \leq b$, then $b * c \leq a * c$,

(vi) $(a \vee b) \wedge(a * b)=b$.

Observe that (iii) implies $b \leq(a * b) * b$.

The relationship between congruences and filters in sectionally pseudocomplemented lattices is illuminated in the next two theorems.

Theorem 2.4 Let $\mathbf{L}=(L, \vee, \wedge, *)$ be a sectionally pseudocomplemented lattice and $\Theta \in$ Con $\mathbf{L}$. Then, $[1] \Theta \in$ Fil $\mathbf{L}$ and for any $x, y \in L$,

$(x, y) \in \Theta$ if and only if $x * y, y * x \in[1] \Theta$,

i.e., $\Phi([1] \Theta)=\Theta$.

Proof Let $a, b \in L$. If $(a, b) \in \Theta$, then $a * b, b * a \in$ $[a * a] \Theta=[1] \Theta$, i.e., $(a, b) \in \Phi([1] \Theta)$. Conversely, if $(a, b) \in \Phi([1] \Theta)$, then $a * b, b * a \in[1] \Theta$ and hence, using (ii) and (iv) of Lemma 2.3,

$a=a \wedge((a * b) * b) \Theta(1 * a) \wedge(1 * b) \Theta((b * a) * a) \wedge b=b$,

i.e., $(a, b) \in \Theta$. This shows $\Phi([1] \Theta)=\Theta$. Due to the substitution property of $\Theta$ with respect to $\vee, \wedge$ and $*$, we 
see that $[1] \Theta$ satisfies the conditions from Definition 2.2 and hence $[1] \Theta \in$ Fil $\mathbf{L}$.

Theorem 2.4 witnesses that sectionally pseudocomplemented lattices are weakly regular.

We can prove also the converse.

Theorem 2.5 Let $\mathbf{L}=(L, \vee, \wedge, *)$ be a sectionally pseudocomplemented lattice and $F \in$ Fil $\mathbf{L}$. Then, $\Phi(F) \in$ Con $\mathbf{L}$ and $[1](\Phi(F))=F$.

Proof Let $a, b, c \in L$. Evidently, $\Phi(F)$ is symmetric and since $1 \in F$ and $x * x \approx 1$ by (i) of Lemma 2.3, it is also reflexive. Assume $a * b, b * a \in F$. Then, by Definition 2.2

$(a * c) *(b * c),(b * c) *(a * c),(c * a) *(c * b)$,

$(c * b) *(c * a),(a \vee c) *(b \vee c),(b \vee c) *(a \vee c)$,

$(a \wedge c) *(b \wedge c),(b \wedge c) *(a \wedge c) \in F$

whence

$(a * c, b * c),(c * a, c * b),(a \vee c, b \vee c),(a \wedge c, b \wedge c) \in \Phi(F)$

Hence, $\Phi(F)$ has the substitution property with respect to all basic operations of $\mathbf{L}$. Since the variety of sectionally pseudocomplemented lattices is congruence permutable, $\Phi(F)$ is also transitive, see, e.g., Werner's Theorem (Werner (1973)) or Corollary 3.1.13 in Chajda et al. (2012), and hence, $\Phi(F) \in$ Con L. Finally, the following are equivalent:

$$
\begin{aligned}
a & \in[1](\Phi(F)), \\
(a, 1) & \in \Phi(F), \\
a * 1,1 * a & \in F, \\
1, a & \in F, \\
a & \in F
\end{aligned}
$$

and hence $[1](\Phi(F))=F$.

It is elementary to check that for every sectionally pseudocomplemented lattice $\mathbf{L}$, (Fil $\mathbf{L}, \subseteq$ ) is a complete lattice.

Example 2.6 The sectionally pseudocomplemented lattice from Fig. 1 has the following filters:

$$
\begin{gathered}
F(1)=\{1\}, \\
F(a)=F(c)=\{a, c, 1\}, \\
F(0)=F(b)=\{0, a, b, c, 1\} .
\end{gathered}
$$

The following corollary follows from Theorems 2.4 and 2.5.

Corollary 2.7 For every sectionally pseudocomplemented lattice $\mathbf{L}$, the mappings $\Phi \mapsto[1] \Phi$ and $F \mapsto \Phi(F)$ are mutually inverse isomorphisms between the complete lattices $(\operatorname{Con} \mathbf{L}, \subseteq)$ and $($ Fil $\mathbf{L}, \subseteq)$.
Let $(L, \vee, \wedge, *)$ be a sectionally pseudocomplemented lattice. A deductive system of $\mathbf{L}$ is a subset $D$ of $L$ containing 1 and satisfying the following condition:

If $a \in D, b \in L$ and $a * b \in D$, then $b \in D$.

In the following $(F *(F * a)) * a$ denotes the set $\{(x *(y * a)) *$ $a \mid x, y \in F\}$. Analogously, we proceed in similar cases.

Theorem 2.8 Let $\mathbf{L}=(L, \vee, \wedge, *)$ be a sectionally pseudocomplemented lattice, $\Theta \in$ Con $\mathbf{L}, F \in$ Fil $\mathbf{L}$ and $a, b \in L$. Then,

(i) Every class of $\Theta$ is a convex subset of $(L, \leq)$,

(ii) $F$ is a deductive system of $\mathbf{L}$,

(iii) $F$ is a lattice filter of $\mathbf{L}$,

(iv) $a *(F \wedge a) \subseteq F$ and $(F *(F * a)) * a \subseteq F$.

Proof (i) If $c, d \in[a] \Theta$ and $c \leq b \leq d$, then

$$
b=c \vee b \in[d \vee b] \Theta=[d] \Theta=[a] \Theta .
$$

(ii) If $a, a * b \in F$, then

$$
b=1 * b \in[a * b](\Phi(F))=[1](\Phi(F))=F .
$$

(iii) If $a \in F$, then

$a \vee b \in[1 \vee b](\Phi(F))=[1](\Phi(F))=F$

Moreover, if $a, b \in F$, then

$a \wedge b \in[1 \wedge 1](\Phi(F))=[1](\Phi(F))=F$.

(iv)

$$
\begin{aligned}
& a *(F \wedge a) \subseteq[a *(1 \wedge a)](\Phi(F)) \\
& =[a * a](\Phi(F))=[1](\Phi(F))=F, \\
& (F *(F * a)) * a \subseteq[(1 *(1 * a)) * a](\Phi(F)) \\
& =[(1 * a) * a](\Phi(F))=[a * a](\Phi(F))= \\
& =[1](\Phi(F))=F .
\end{aligned}
$$

\section{Sectionally pseudocomplemented posets}

Now we turn our attention to sectionally pseudocomplemented posets. 
Definition 3.1 Let $\mathbf{P}=(P, \leq)$ be a poset. Then, $\mathbf{P}$ is called sectionally pseudocomplemented if for all $a, b \in P$ there exists a greatest element $c$ of $P$ satisfying

$L(U(a, b), c)=L(b)$.

This element $c$ is called the sectional pseudocomplement $a * b$ of $a$ with respect to $b$. We write sectionally pseudocomplemented posets in the form $(P, \leq, *)$. A strongly sectionally pseudocomplemented poset is an ordered quadruple $(P, \leq$ $, *, 1)$ such that $(P, \leq, *)$ is a sectionally pseudocomplemented poset with greatest element 1 satisfying the identity

$x \leq(x * y) * y$.

The following results were proved in Chajda et al. (2021).

Lemma 3.2 If $\mathbf{P}=(P, \leq, *)$ is a sectionally pseudocomplemented poset with greatest element 1 and $a, b, c \in P$, then

(i) $a * b=1$ if and only if $a \leq b$,

(ii) $1 * a=a$,

(iii) $a \leq b * a$,

(iv) if $b \leq a$, then $a \leq(a * b) * b$,

(v) if $a \leq b$, then $b * c \leq a * c$,

(vi) $L(U(a, b), a * b)=L(b)$.

Observe that (iii) implies $b \leq(a * b) * b$. Hence, in case $a \leq b$ we have $a \leq(a * b) * b$.

It is easy to see that every sectionally pseudocomplemented lattice is a strongly sectionally pseudocomplemented poset, and a lattice is sectionally pseudocomplemented if and only if it is sectionally pseudocomplemented as a poset.

Remark 3.3 If $(P, \leq, *)$ is a sectionally pseudocomplemented poset and $a, b \in P$, then

$L(U(a, b), a * b)=L(b)$

which shows that there exists the infimum $U(a, b) \wedge(a * b)$, and hence, the previous is equivalent to

$U(a, b) \wedge(a * b)=b$.

Thus, in case $a \geq b$ we obtain $a \wedge(a * b)=b$.

An example of a strongly sectionally pseudocomplemented poset which is not a lattice is visualized in Fig. 2.
Fig. 2 Strongly sectionally pseudocomplemented poset

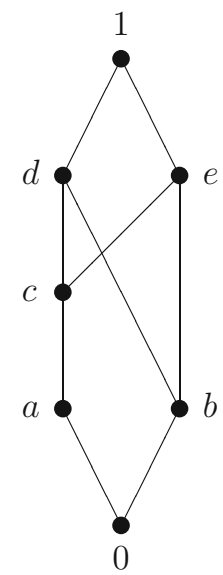

The operation table of $*$ is as follows:

\begin{tabular}{l|lllllll}
$*$ & 0 & $a$ & $b$ & $c$ & $d$ & $e$ & 1 \\
\hline 0 & 1 & 1 & 1 & 1 & 1 & 1 & 1 \\
$a$ & $b$ & 1 & $b$ & 1 & 1 & 1 & 1 \\
$b$ & $c$ & $a$ & 1 & $c$ & 1 & 1 & 1 \\
$c$ & $b$ & $a$ & $b$ & 1 & 1 & 1 & 1 \\
$d$ & 0 & $a$ & $b$ & $c$ & 1 & $e$ & 1 \\
$e$ & 0 & $a$ & $b$ & $c$ & $d$ & 1 & 1 \\
1 & 0 & $a$ & $b$ & $c$ & $d$ & $e$ & 1
\end{tabular}

This poset is not relatively pseudocomplemented since the relative pseudocomplement of $c$ with respect to $a$ does not exist.

It should be noted that there are sectionally pseudocomplemented posets which are not strongly sectionally pseudocomplemented, see, e.g., Chajda et al. (2021), but these are rather curious.

Since a sectionally pseudocomplemented poset $\mathbf{P}$ has only one operation, namely $*$, a congruence on $\mathbf{P}$ should satisfy the substitution property with respect to $*$. However, this condition is rather weak and we cannot expect to obtain a natural relationship between congruences and congruence kernels similar to that obtained for sectionally pseudocomplemented lattices in the previous section. Namely, our concept of a congruence on a strongly sectionally pseudocomplemented poset should respect also some aspects of the partial order relation. This is the reason why we introduce the following property.

Definition 3.4 A binary relation $\rho$ on a poset is called minstable if the following holds: If $(a, b),(c, d) \in \rho, a$ is comparable with $c$ and $b$ is comparable with $d$, then

$(\min (a, c), \min (b, d)) \in \rho$.

Observe that this condition trivially holds if $a \leq c$ and $b \leq d$ or if $a \geq c$ and $b \geq d$.

Now we can define 
Definition 3.5 Let $\mathbf{P}=(P, \leq, *)$ be a sectionally pseudocomplemented poset. A congruence on $\mathbf{P}$ is a min-stable congruence on the algebraic reduct $(P, *)$ of $\mathbf{P}$. Let Con $\mathbf{P}$ denote the set of all congruences on $\mathbf{P}$.

Note that the congruences on a sectionally pseudocomplemented lattice $\mathbf{L}$ may not coincide with the congruences on $\mathbf{L}$ if it is considered only as a sectionally pseudocomplemented poset.

In analogy to the lattice case, we define

Definition 3.6 Let $\mathbf{P}=(P, \leq, *, 1)$ be a sectionally pseudocomplemented poset with greatest element 1. A filter of $\mathbf{P}$ is a subset $F$ of $P$ containing 1 and satisfying the following conditions for all $x, y, z, v \in P$ :

- If $x * y, y * x \in F$, then $(x * z) *(y * z),(z * x) *(z * y) \in F$,

- if $x * y, y * x, z * v, v * z \in F, x$ and $z$ are comparable and $y$ and $v$ are comparable, then $\min (x, z) * \min (y, v) \in F$.

Let Fil $\mathbf{P}$ denote the set of all filters of $\mathbf{P}$. It is elementary to check that for every strongly sectionally pseudocomplemented poset $\mathbf{P},(\operatorname{Con} \mathbf{P}, \subseteq)$ and (Fil $\mathbf{P}, \subseteq)$ are complete lattices. For any subset $M$ of $P$ put

$\Phi(M):=\left\{(x, y) \in P^{2} \mid x * y, y * x \in M\right\}$.

The relationship between congruences and filters in strongly sectionally pseudocomplemented posets is illuminated in the next two theorems.

Theorem 3.7 Let $\mathbf{P}=(P, \leq, *, 1)$ be a strongly sectionally pseudocomplemented poset and $\Theta \in$ Con $\mathbf{L}$. Then, $[1] \Theta \in$ Fil $\mathbf{L}$ and for any $x, y \in P$,

$(x, y) \in \Theta$ if and only if $x * y, y * x \in[1] \Theta$,

i.e., $\Phi([1] \Theta)=\Theta$.

Proof Let $a, b \in L$. If $(a, b) \in \Theta$, then, by Lemma 3.2, $a * b, b * a \in[a * a] \Theta=[1] \Theta$, i.e., $(a, b) \in \Phi([1] \Theta)$. Conversely, if $(a, b) \in \Phi([1] \Theta)$, then $a * b, b * a \in[1] \Theta$ and hence, using again Lemma 3.2,

$(a,(b * a) * a)=(1 * a,(b * a) * a) \in \Theta$,

$((a * b) * b, b)=((a * b) * b, 1 * b) \in \Theta$.

Since $\mathbf{P}$ is strongly sectionally pseudocomplemented, we have $a \leq(a * b) * b$ and $(b * a) * a \geq b$; thus, by minstability of $\Theta$ we conclude

$(a, b)=(\min (a,(a * b) * b), \min ((b * a) * a, b)) \in \Theta$.

This shows $\Phi([1] \Theta)=\Theta$. Due to the substitution property of $\Theta$ with respect to $*$ and the min-stability of $\Theta$, we obtain $[1] \Theta \in$ Fil L.
We have shown that every congruence $\Theta$ on a strongly sectionally pseudocomplemented poset is fully determined by its 1-class [1] $\Theta$. Hence, we conclude

Corollary 3.8 Strongly sectionally pseudocomplemented posets are weakly regular.

We can prove also the converse.

Theorem 3.9 Let $\mathbf{P}=(P, \leq, *, 1)$ be a strongly sectionally pseudocomplemented poset and $F \in$ Fil $\mathbf{P}$. Then, $\Phi(F) \in$ Con $\mathbf{P}$ and $[1](\Phi(F))=F$.

Proof Let $a, b, c, d \in P$. Evidently, $\Phi(F)$ is symmetric and since $1 \in F$ and $x * x \approx 1$, it is also reflexive. If $(a, b) \in$ $\Phi(F)$, then $a * b, b * a \in F$ and hence, using the properties listed in Definition 3.6,

$(a * c) *(b * c),(b * c) *(a * c) \in F$,

$(c * a) *(c * b),(c * b) *(c * a) \in F$.

Thus, $(a * c, b * c),(c * a, c * b) \in \Phi(F)$. Hence, $\Phi(F)$ has the substitution property with respect to $*$. Moreover, if $(a, b),(c, d) \in \Phi(F), a$ is comparable with $c$ and $b$ is comparable with $d$, then $a * b, b * a, c * d, d * c \in F$ and by Definition 3.6

$\min (a, c) * \min (b, d), \min (b, d) * \min (a, c) \in F$,

i.e., $(\min (a, c), \min (b, d)) \in \Phi(F)$. This shows that $\Phi(F)$ is min-stable. If $(a, b),(b, c) \in \Phi(F)$, then

$(a * b) * b \Phi(F)(b * b) * c=1 * c=c$,

$a=1 * a=(b * b) * a \Phi(F)(c * b) * b$

and hence using min-stability of $\Phi(F)$

$(a, c)=(\min ((a * b) * b, a), \min (c,(c * b) * b)) \in \Phi(F)$,

i.e., $\Phi(F)$ is transitive. Therefore, $\Phi(F) \in$ Con $\mathbf{P}$. Finally, the following are equivalent:

$$
\begin{aligned}
a & \in[1](\Phi(F)), \\
(a, 1) & \in \Phi(F), \\
a * 1,1 * a & \in F, \\
1, a & \in F, \\
a & \in F .
\end{aligned}
$$

This shows $[1](\Phi(F))=F$.

Example 3.10 The lattice of filters of the strongly sectionally pseudocomplemented poset from Fig. 2 consists of the following six filters:

$$
F(1)=\{1\},
$$


Fig. 3 Lattice of filters of the poset from Fig. 2

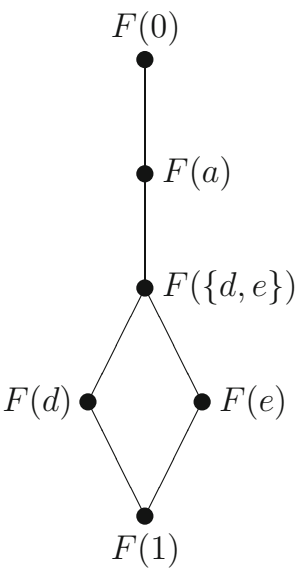

$$
\begin{aligned}
F(d) & =\{d, 1\}, \\
F(e) & =\{e, 1\}, \\
F(\{d, e\}) & =\{d, e, 1\}, \\
F(a)=F(c) & =\{a, c, d, e, 1\}, \\
F(0)=F(b) & =\{0, a, b, c, d, e, 1\} .
\end{aligned}
$$

The corresponding Hasse diagram is depicted in Fig. 3:

The following corollary follows from Theorems 3.7 and 3.9 .

Corollary 3.11 For every strongly sectionally pseudocomplemented poset $\mathbf{P}$, the mappings $\Phi \mapsto[1] \Phi$ and $F \mapsto \Phi(F)$ are mutually inverse isomorphisms between the complete lattices (Con $\mathbf{P}, \subseteq)$ and (Fil $\mathbf{P}, \subseteq)$.

\section{Properties of filters}

Using the min-stability property of congruences in strongly sectionally pseudocomplemented posets, we can prove

Theorem 4.1 Let $\mathbf{P}=(P, \leq, *, 1)$ be a strongly sectionally pseudocomplemented poset and $\Theta \in$ Con $\mathbf{P}$. Then, every class of $\Theta$ is a convex subset of $(P, \leq)$.

Proof If $a, c \in P, b, d \in[a] \Theta$ and $b \leq c \leq d$, then

$(c * d) * b=1 * b=b \leq c \leq(c * b) * b$,

$((c * d) * b,(c * b) * b) \in \Theta$

and hence by min-stability of $\Theta$ we obtain

$(b, c)=(\min ((c * d) * b, c), \min ((c * b) * b, c)) \in \Theta$,

which implies $c \in[b] \Theta=[a] \Theta$.

We now investigate quotients $\mathbf{P} / \Theta$ of strongly sectionally pseudocomplemented posets $\mathbf{P}$ with respect to its congruences.
Let $\mathbf{P}=(P, \leq, *, 1)$ be a strongly sectionally pseudocomplemented poset and $\Theta \in$ Con $\mathbf{P}$. We define a binary relation $\leq^{\prime}$ on $P / \Theta$ by

for all $a, b \in P,[a] \Theta \leq^{\prime}[b] \Theta$ if and only if $[a] \Theta *[b] \Theta=[1] \Theta$.

Recall that a poset $(P, \leq)$ is called up-directed if for any $x, y \in P$ there exists some $z \in P$ with $x, y \leq z$. Hence, every poset having a greatest element is up-directed.

It should be mentioned that the poset $\left(P / \Theta, \leq^{\prime}\right)$ where $\mathbf{P}=(P, \leq, *, 1)$ denotes the strongly sectionally pseudocomplemented poset from Fig. 2 and $\Theta$ the congruence on $\mathbf{P}$ corresponding to the filter $F(\{d, e\})$ of $\mathbf{P}$ is isomorphic to the lattice from Fig. 1.

The following theorem was partly proved for congruences on the algebraic reduct $(P, *)$ in Chajda et al. (2021).

Theorem 4.2 Let $\mathbf{P}=(P, \leq, *, 1)$ be a strongly sectionally pseudocomplemented poset, $n \geq 1, a, a_{1}, \ldots, a_{n}, b \in P$ and $\Theta \in$ Con $\mathbf{P}$. Then, the following hold:

(i) if $a \leq b$, then $[a] \Theta \leq^{\prime}[b] \Theta$,

(ii) $[a] \Theta \leq^{\prime}[b] \Theta$ if and only if there exists some $c \in[b] \Theta$ with $a \leq c$,

(iii) $\left(P / \Theta, \leq^{\prime}\right)$ is a poset,

(iv) Every class of $\Theta$ is up-directed,

(v) $U\left(\left[a_{1}\right] \Theta, \ldots,\left[a_{n}\right] \Theta\right)=\left\{[x] \Theta \mid x \in U\left(a_{1}, \ldots, a_{n}\right)\right\}$ in $\left(P / \Theta, \leq^{\prime}\right)$.

Proof (i) If $a \leq b$, then $a * b=1$ whence $a * b \Theta 1$, i.e., $[a] \Theta *[b] \Theta=[a * b] \Theta=[1] \Theta$; thus, $[a] \Theta \leq^{\prime}[b] \Theta$.

(ii) If $[a] \Theta \leq^{\prime}[b] \Theta$, then $a * b \Theta 1$ and hence $a \leq(a * b) *$ $b \in[1 * b] \Theta=[b] \Theta$. So one can put $c:=(a * b) * b$. If, conversely, there exists some $c \in[b] \Theta$ with $a \leq c$, then according to (i) we have $[a] \Theta \leq^{\prime}[c] \Theta=[b] \Theta$.

(iii) Obviously, $\leq^{\prime}$ is reflexive. Now assume $[a] \Theta \leq^{\prime}[b] \Theta$ and $[b] \Theta \leq^{\prime}[a] \Theta$. Then, by (ii), there exists some $c \in[b] \Theta$ with $a \leq c$. Because of $[c] \Theta=[b] \Theta \leq^{\prime}$ $[a] \Theta$ there exists some $d \in[a] \Theta$ with $c \leq d$. Since $a \leq c \leq d, a, d \in[a] \Theta$ and $\left([a] \Theta, \leq^{\prime}\right)$ is convex we conclude $c \in[a] \Theta$. Therefore $[a] \Theta=[c] \Theta=[b] \Theta$ which proves antisymmetry of $\leq^{\prime}$. Finally, let $c \in P$ and assume $[a] \Theta \leq^{\prime}[b] \Theta$ and $[b] \Theta \leq^{\prime}[c] \Theta$. Then, by (ii) there exists some $e \in[b] \Theta$ with $a \leq e$ and because of $[e] \Theta=[b] \Theta \leq^{\prime}[c] \Theta$ some $f \in[c] \Theta$ with $e \leq f$. From $a \leq e \leq f$ we have $a \leq f$ which implies $[a] \Theta \leq^{\prime}[f] \Theta=[c] \Theta$ by (i), proving transitivity of $\leq$.

(iv) Let $b, c \in[a] \Theta$. Then,

$(b * c) * c \in[(c * c) * c] \Theta=[1 * c] \Theta=[c] \Theta=[a] \Theta$, $b \leq(b * c) * c$ since $\mathbf{P}$ is strongly sectionally pseudocomplemented, 
$c \leq(b * c) * c$ according to Lemma 3.2 (iii).

Thus, $(b * c) * c$ is a common upper bound of $b$ and $c$ within $([a] \Theta, \leq)$.

(v) Assume $[a] \Theta \in U\left(\left[a_{1}\right] \Theta, \ldots,\left[a_{n}\right] \Theta\right)$. According to (ii), for all $i \in\{1, \ldots, n\}$ there exists some $b_{i} \in[a] \Theta$ with $a_{i} \leq b_{i}$. Because of (iv), $\left([a] \Theta, \leq^{\prime}\right)$ is updirected and hence there exists some $c \in[a] \Theta$ with $b_{1}, \ldots, b_{n} \leq c$. This shows

$[a] \Theta=[c] \Theta \in\left\{[x] \Theta \mid x \in U\left(a_{1}, \ldots, a_{n}\right)\right\}$.

The converse inclusion follows from (i).

From (iv) we conclude that if $(P, \leq)$ satisfies the ascending chain condition (in particular, if $P$ is finite), then every class of $\Theta$ has a greatest element.

The following concept is inspired by the derivation rule Modus Ponens in the non-classical logic based on a sectionally pseudocomplemented poset where $*$ models the logical connective implication.

Let $(P, \leq, *, 1)$ be a strongly sectionally pseudocomplemented poset. A deductive system of $\mathbf{P}$ is a subset $D$ of $P$ containing 1 and satisfying the following condition:

If $a \in D, b \in P$ and $a * b \in D$, then $b \in D$.

We can prove the following result in analogy to the corresponding result for sectionally pseudocomplemented lattices.

Theorem 4.3 Let $\mathbf{P}=(P, \leq, *, 1)$ be a strongly sectionally pseudocomplemented poset, $F \in$ Fil $\mathbf{P}$ and $c \in P$. Then,

(i) $F$ is a deductive system of $\mathbf{P}$,

(ii) $F$ is an order filter of $\mathbf{P}$,

(iii) $P * F \subseteq F$,

(iv) $c *(F \wedge c),(F *(F * c)) * c \subseteq F$.

Proof We use the fact that the filter $F$ is the 1-class of the congruence $\Phi(F)$.

(i) If $a \in F, b \in P$ and $a * b \in F$, then

$$
b=1 * b \in[a * b](\Phi(F))=[1](\Phi(F))=F .
$$

(ii) If $a \in F, b \in P$ and $a \leq b$, then $a * b=1 \in F$ and hence $b \in F$ by (i).

(iii) If $a \in P$ and $b \in F$, then $a * b \in[a * 1](\Phi(F))=$ $[1](\Phi(F))=F$.

(iv)

$$
c *(F \wedge c) \subseteq[c *(1 \wedge c)](\Phi(F))
$$

$$
\begin{aligned}
& =[c * c](\Phi(F))=[1](\Phi(F))=F \\
& (F *(F * c)) * c \subseteq[(1 *(1 * c)) * c](\Phi(F)) \\
& =[(1 * c) * c](\Phi(F))=[c * c](\Phi(F))= \\
& =[1](\Phi(F))=F .
\end{aligned}
$$

Theorem 4.3 shows that every filter is a deductive system. However, our concept of a filter is rather complicated and it seems that not all the properties of a filter are necessary to prove this assertion. We can prove

Proposition 4.4 Let $\mathbf{P}=(P, \leq, *, 1)$ be a strongly sectionally pseudocomplemented poset and $M$ a subset of $P$ containing 1 and satisfying $(M *(M * x)) * x \subseteq M$ for all $x \in P$. Then, $M$ is a deductive system of $\mathbf{P}$.

Proof Let $a \in M$ and $b \in P$. We have $1 \in M$. If $a \leq b$, then

$b=1 * b=(a * b) * b=(a *(1 * b)) * b \in(M *(M * b)) * b \subseteq M$.

Hence, if $a * b \in M$, then because of $a \leq(a * b) * b$ we have $(a * b) * b \in M$ which implies

$b=1 * b=(((a * b) * b) *((a * b) * b)) * b \in(M *(M * b)) * b \subseteq M$.

Observe that the condition mentioned in Proposition 4.4 is just the second one of (iv) of Theorem 4.3.

For the concept of an ideal of a universal algebra which corresponds to our concept of a filter and for the concept of ideal terms, the reader is referred to Ursini (1972). In particular, for ideals (alias filters) in permutable and weakly regular varieties see also Chajda et al. (2012) for details.

Definition 4.5 An ideal term for sectionally pseudocomplemented lattices is a term $t\left(x_{1}, \ldots, x_{n}, y_{1}, \ldots, y_{m}\right)$ in the language of sectionally pseudocomplemented lattices satisfying the identity

$t\left(x_{1}, \ldots, x_{n}, 1, \ldots, 1\right) \approx 1$

Of course, there exist an infinite number of ideal terms in sectionally pseudocomplemented lattices. The following list including five ideal terms is a so-called basis for filters in sectionally pseudocomplemented lattices, i.e., filters can be characterized by this short list of ideal terms.

Lemma 4.6 The following terms are ideal terms for sectionally pseudocomplemented lattices:

$t_{1}:=1$,

$t_{2}\left(x_{1}, x_{2}, x_{3}, y_{1}, y_{2}\right)$ 


$$
\begin{aligned}
& :=\left(\left(\left(x_{1} \vee x_{2}\right) \wedge\left(y_{1} * x_{2}\right) \wedge y_{2}\right) \vee x_{3}\right) \\
& *\left(x_{2} \vee x_{3}\right), \\
& t_{3}\left(x_{1}, x_{2}, x_{3}, y_{1}, y_{2}\right) \\
& :=\left(\left(\left(x_{1} \vee x_{2}\right) \wedge\left(y_{1} * x_{2}\right) \wedge y_{2}\right) \wedge x_{3}\right) \\
& *\left(x_{2} \wedge x_{3}\right), \\
& t_{4}\left(x_{1}, x_{2}, x_{3}, y_{1}, y_{2}\right) \\
& :=\left(\left(\left(x_{1} \vee x_{2}\right) \wedge\left(y_{1} * x_{2}\right) \wedge y_{2}\right) * x_{3}\right) \\
& *\left(x_{2} * x_{3}\right), \\
& t_{5}\left(x_{1}, x_{2}, x_{3}, y_{1}, y_{2}\right) \\
& :=\left(x_{3} * x_{1}\right) *\left(x_{3} *\left(\left(x_{1} \vee x_{2}\right) \wedge\left(y_{2} * x_{1}\right) \wedge y_{1}\right)\right) .
\end{aligned}
$$

\section{Proof Put}

$t(x, y, z, u):=(x \vee y) \wedge(z * y) \wedge u$.

Then,

$$
\begin{aligned}
& t_{2}\left(x_{1}, x_{2}, x_{3}, y_{1}, y_{2}\right)=\left(t\left(x_{1}, x_{2}, y_{1}, y_{2}\right) \vee x_{3}\right) *\left(x_{2} \vee x_{3}\right), \\
& t_{3}\left(x_{1}, x_{2}, x_{3}, y_{1}, y_{2}\right)=\left(t\left(x_{1}, x_{2}, y_{1}, y_{2}\right) \wedge x_{3}\right) *\left(x_{2} \wedge x_{3}\right), \\
& t_{4}\left(x_{1}, x_{2}, x_{3}, y_{1}, y_{2}\right)=\left(t\left(x_{1}, x_{2}, y_{1}, y_{2}\right) * x_{3}\right) *\left(x_{2} * x_{3}\right), \\
& t_{5}\left(x_{1}, x_{2}, x_{3}, y_{1}, y_{2}\right)=\left(x_{3} * x_{1}\right) *\left(x_{3} * t\left(x_{2}, x_{1}, y_{2}, y_{1}\right)\right) .
\end{aligned}
$$

and according to Lemma 2.3

$$
\begin{aligned}
& t(x, y, 1,1)=(x \vee y) \wedge(1 * y) \wedge 1=(x \vee y) \wedge y=y \\
& \text { and hence } \\
& t_{2}\left(x_{1}, x_{2}, x_{3}, 1,1\right) \\
& \quad=\left(t\left(x_{1}, x_{2}, 1,1\right) \vee x_{3}\right) *\left(x_{2} \vee x_{3}\right)=\left(x_{2} \vee x_{3}\right) \\
& \quad *\left(x_{2} \vee x_{3}\right)=1, \\
& t_{3}\left(x_{1}, x_{2}, x_{3}, 1,1\right) \\
& \quad=\left(t\left(x_{1}, x_{2}, 1,1\right) \wedge x_{3}\right) *\left(x_{2} \wedge x_{3}\right)=\left(x_{2} \wedge x_{3}\right) \\
& \quad *\left(x_{2} \wedge x_{3}\right)=1, \\
& \quad t_{4}\left(x_{1}, x_{2}, x_{3}, 1,1\right)=\left(t\left(x_{1}, x_{2}, 1,1\right) * x_{3}\right) *\left(x_{2} * x_{3}\right) \\
& \quad=\left(x_{2} * x_{3}\right) *\left(x_{2} * x_{3}\right)=1, \\
& t_{5}\left(x_{1}, x_{2}, x_{3}, 1,1\right)=\left(x_{3} * x_{1}\right) *\left(x_{3} * t\left(x_{2}, x_{1}, 1,1\right)\right) \\
& \quad=\left(x_{3} * x_{1}\right) *\left(x_{3} * x_{1}\right)=1 .
\end{aligned}
$$

The closedness with respect to ideal terms was also introduced by A. Ursini ( Ursini (1972)).

Definition 4.7 A subset $A$ of a sectionally pseudocomplemented lattice $\mathbf{L}=(L, \vee, \wedge, *)$ is said to be closed with respect to the ideal terms $t_{i}\left(x_{1}, \ldots, x_{n}, y_{1}, \ldots, y_{m}\right), i \in I$, if for every $i \in I$, all $x_{1}, \ldots, x_{n} \in L$ and all $y_{1}, \ldots, y_{m} \in A$ we have $t_{i}\left(x_{1}, \ldots, x_{n}, y_{1}, \ldots, y_{m}\right) \in A$.
Now we prove that the ideal terms listed in Lemma 4.6 form a basis for filters, i.e., filters are characterized as those subsets which are closed with respect to these ideal terms.

Theorem 4.8 Let $\mathbf{L}=(L, \vee, \wedge, *)$ be a sectionally pseudocomplemented lattice and $F \subseteq L$. Then, $F \in$ Fil $\mathbf{L}$ if and only if $F$ is closed with respect to the ideal terms $t_{1}, \ldots, t_{5}$ listed in Lemma 4.6.

Proof If $F \in$ Fil $\mathbf{L}$, then $F=[1](\Phi(F))$ according to Theorem 2.5 , and if

$t_{i}\left(x_{1}, \ldots, x_{n}, y_{1}, \ldots, y_{m}\right), \quad i \in\{1, \ldots, 5\}$,

are the ideal terms listed in Lemma 4.6, $a_{1}, \ldots, a_{n} \in L$ and $b_{1}, \ldots, b_{m} \in F$, then

$$
\begin{aligned}
& t_{i}\left(a_{1}, \ldots, a_{n}, b_{1}, \ldots, b_{m}\right) \in\left[t_{i}\left(a_{1}, \ldots, a_{n}, 1, \ldots, 1\right)\right](\Phi(F)) \\
& \quad=[1](\Phi(F))=F
\end{aligned}
$$

according to Lemma 4.6 and hence $F$ is closed with respect to the ideal terms $t_{1}, \ldots, t_{5}$. Conversely, assume $F$ to be closed with respect to the ideal terms $t_{1}, \ldots, t_{5}$. Then, $1=t_{1} \in F$. Now assume $a, b \in L$ and $a * b, b * a \in F$. For the term

$t(x, y, z, u):=(x \vee y) \wedge(z * y) \wedge u$,

we have

$t_{2}\left(x_{1}, x_{2}, x_{3}, y_{1}, y_{2}\right)=\left(t\left(x_{1}, x_{2}, y_{1}, y_{2}\right) \vee x_{3}\right) *\left(x_{2} \vee x_{3}\right)$,

$t_{3}\left(x_{1}, x_{2}, x_{3}, y_{1}, y_{2}\right)=\left(t\left(x_{1}, x_{2}, y_{1}, y_{2}\right) \wedge x_{3}\right) *\left(x_{2} \wedge x_{3}\right)$,

$t_{4}\left(x_{1}, x_{2}, x_{3}, y_{1}, y_{2}\right)=\left(t\left(x_{1}, x_{2}, y_{1}, y_{2}\right) * x_{3}\right) *\left(x_{2} * x_{3}\right)$,

$t_{5}\left(x_{1}, x_{2}, x_{3}, y_{1}, y_{2}\right)=\left(x_{3} * x_{1}\right) *\left(x_{3} * t\left(x_{2}, x_{1}, y_{2}, y_{1}\right)\right)$

and according to Lemma 2.3 (iv) and (vi) we obtain

$$
\begin{aligned}
& t(x, y, x * y, y * x)=(x \vee y) \wedge((x * y) * y) \wedge(y * x)= \\
& \quad=((y \vee x) \wedge(y * x)) \wedge((x * y) * y) \\
& \quad=x \wedge((x * y) * y)=x .
\end{aligned}
$$

Hence,

$$
\begin{aligned}
& (a \vee c) *(b \vee c)=(t(a, b, a * b, b * a) \vee c) *(b \vee c) \\
& \quad=t_{2}(a, b, c, a * b, b * a) \in F, \\
& (a \wedge c) *(b \wedge c)=(t(a, b, a * b, b * a) \wedge c) *(b \wedge c) \\
& \quad=t_{3}(a, b, c, a * b, b * a) \in F \\
& (a * c) *(b * c)=(t(a, b, a * b, b * a) * c) *(b * c) \\
& \quad=t_{4}(a, b, c, a * b, b * a) \in F \\
& (c * a) *(c * b)=(c * a) *(c * t(b, a, b * a, a * b)) \\
& \quad=t_{5}(a, b, c, a * b, b * a) \in F
\end{aligned}
$$

showing $F \in$ Fil L. 
Remark 4.9 Let us note that the term $t$ from the proof of Theorem 4.8 gives rise to a Maltsev term. Namely, if

$$
\begin{aligned}
t(x, y, z, u) & :=(x \vee y) \wedge(z * y) \wedge u \text { and } \\
q(x, y, z) & :=t(x, z, x * y, y * x),
\end{aligned}
$$

then

$$
\begin{aligned}
q(x, y, z) & =(x \vee z) \wedge((x * y) * z) \wedge(y * x), \\
q(x, x, z) & =(x \vee z) \wedge((x * x) * z) \wedge(x * x) \\
& =(x \vee z) \wedge(1 * z) \wedge 1=(x \vee z) \wedge z=z, \\
q(x, z, z) & =(x \vee z) \wedge((x * z) * z) \wedge(z * x) \\
& =((z \vee x) \wedge(z * x)) \wedge((x * z) * z)= \\
& =x \wedge((x * z) * z)=x .
\end{aligned}
$$

Observe that the Maltsev term $q(x, y, z)$ is different from that in Proposition 2.1.

In the following we write $a \wedge b \wedge c$ instead of $\inf (a, b, c)$. Now we introduce a certain modification of the notion an ideal term (for posets) which need not be defined everywhere. This will be used in the sequel.

Definition 4.10 A partial ideal term for sectionally pseudocomplemented posets with greatest element 1 is a partially defined term $T\left(x_{1}, \ldots, x_{n}, y_{1}, \ldots, y_{m}\right)$ in the language of sectionally pseudocomplemented posets with greatest element 1 satisfying the identity

$T\left(x_{1}, \ldots, x_{n}, 1, \ldots, 1\right) \approx 1$.

This language contains also a binary operator $U(x, y)$.

Using the concept of partial ideal terms, we will try to describe filters also in strongly sectionally pseudocomplemented posets. Similarly as in Lemma 4.6, we firstly get a list of four partial ideal terms which will be shown to suffice.

Lemma 4.11 The following partial terms are partial ideal terms for strongly sectionally pseudocomplemented posets:

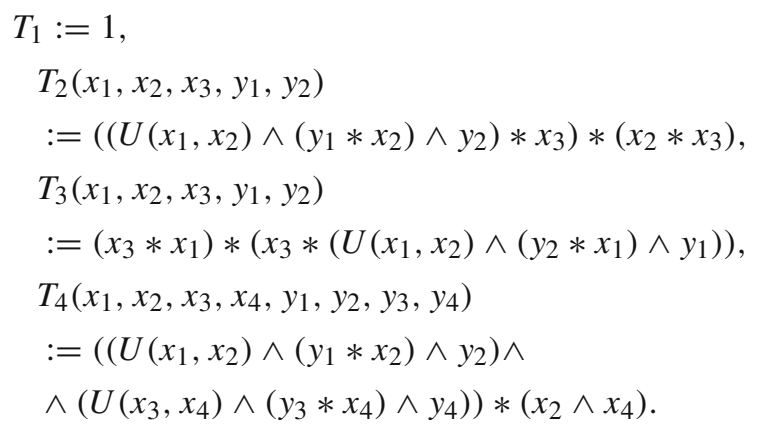

Proof Put

$T(x, y, z, u):=U(x, y) \wedge(z * y) \wedge u$.

Then,

$$
\begin{aligned}
& T_{2}\left(x_{1}, x_{2}, x_{3}, y_{1}, y_{2}\right) \\
& \quad=\left(T\left(x_{1}, x_{2}, y_{1}, y_{2}\right) * x_{3}\right) \\
& \quad *\left(x_{2} * x_{3}\right) \\
& \quad T_{3}\left(x_{1}, x_{2}, x_{3}, y_{1}, y_{2}\right) \\
& \quad=\left(x_{3} * x_{1}\right) \\
& \quad *\left(x_{3} * T\left(x_{2}, x_{1}, y_{2}, y_{1}\right)\right) \\
& \quad T_{4}\left(x_{1}, x_{2}, x_{3}, x_{4}, y_{1}, y_{2}, y_{3}, y_{4}\right) \\
& \quad=\left(T\left(x_{1}, x_{2}, y_{1}, y_{2}\right) \wedge T\left(x_{3}, x_{4}, y_{3}, y_{4}\right)\right) \\
& \quad *\left(x_{2} \wedge x_{4}\right)
\end{aligned}
$$

and according to Lemma 3.2

$T(x, y, 1,1)=U(x, y) \wedge(1 * y) \wedge 1=U(x, y) \wedge y=y$.

Hence,

$$
\begin{aligned}
& T_{2}\left(x_{1}, x_{2}, x_{3}, 1,1\right) \\
& \quad=\left(T\left(x_{1}, x_{2}, 1,1\right) * x_{3}\right) \\
& \quad *\left(x_{2} * x_{3}\right)=\left(x_{2} * x_{3}\right) *\left(x_{2} * x_{3}\right)=1, \\
& T_{3}\left(x_{1}, x_{2}, x_{3}, 1,1\right) \\
& \quad=\left(x_{3} * x_{1}\right) *\left(x_{3} * T\left(x_{2}, x_{1}, 1,1\right)\right)=\left(x_{3} * x_{1}\right) \\
& \quad *\left(x_{3} * x_{1}\right)=1, \\
& T_{4}\left(x_{1}, x_{2}, x_{3}, x_{4}, 1,1,1,1\right) \\
& =\left(T\left(x_{1}, x_{2}, 1,1\right) \wedge T\left(x_{3}, x_{4}, 1,1\right)\right) \\
& \quad *\left(x_{2} \wedge x_{4}\right)= \\
& \quad=\left(x_{2} \wedge x_{4}\right) *\left(x_{2} \wedge x_{4}\right)=1 .
\end{aligned}
$$

Now we define closedness with respect to partial ideal terms.

Definition 4.12 A subset A of a strongly sectionally pseudocomplemented poset $\mathbf{P}=(P, \leq, *, 1)$ is said to be closed with respect to the partial ideal terms $T_{i}\left(x_{1}, \ldots, x_{n}, y_{1}, \ldots\right.$ $\left.\ldots, y_{m}\right), i \in I$, if for every $i \in I$, all $x_{1}, \ldots, x_{n} \in P$ and all $y_{1}, \ldots, y_{m} \in A$ we have that $T_{i}\left(x_{1}, \ldots, x_{n}, y_{1}, \ldots, y_{m}\right)$ is defined and $T_{i}\left(x_{1}, \ldots, x_{n}, y_{1}, \ldots, y_{m}\right) \in A$.

Although our ideal terms are only partial, we can prove that every subset of a strongly sectionally pseudocomplemented poset $\mathbf{P}$ closed with respect to them is really a filter of $\mathbf{P}$.

Theorem 4.13 Let $\mathbf{P}=(P, \leq, *, 1)$ be a strongly sectionally pseudocomplemented poset and $F$ a subset of $P$ that is closed 
with respect to the partial ideal terms $T_{1}, \ldots, T_{4}$ listed in Lemma 4.11. Then, $F \in$ Fil P.

Proof We have $1=T_{1} \in F$. Now assume $a, b, c, d \in P$ and $a * b, b * a, c * d, d * c \in F$. For the partial term

$T(x, y, z, u):=U(x, y) \wedge(z * y) \wedge u$,

we have

$$
\begin{aligned}
& T_{2}\left(x_{1}, x_{2}, x_{3}, y_{1}, y_{2}\right) \\
& \quad=\left(T\left(x_{1}, x_{2}, y_{1}, y_{2}\right) * x_{3}\right) *\left(x_{2} * x_{3}\right), \\
& T_{3}\left(x_{1}, x_{2}, x_{3}, y_{1}, y_{2}\right) \\
& \quad=\left(x_{3} * x_{1}\right) *\left(x_{3} * T\left(x_{2}, x_{1}, y_{2}, y_{1}\right)\right), \\
& T_{4}\left(x_{1}, x_{2}, x_{3}, x_{4}, y_{1}, y_{2}, y_{3}, y_{4}\right) \\
& =\left(T\left(x_{1}, x_{2}, y_{1}, y_{2}\right) \wedge T\left(x_{3}, x_{4}, y_{3}, y_{4}\right)\right) *\left(x_{2} \wedge x_{4}\right)
\end{aligned}
$$

and according to Lemma 3.2 and Remark 3.3 we obtain

$$
\begin{aligned}
T(x, y, x * y, y * x) & =U(x, y) \wedge((x * y) * y) \wedge(y * x)= \\
& =(U(y, x) \wedge(y * x)) \wedge((x * y) * y) \\
& =x \wedge((x * y) * y)=x .
\end{aligned}
$$

Hence,

$$
\begin{aligned}
(a * c) *(b * c) & =(T(a, b, a * b, b * a) * c) *(b * c) \\
& =T_{2}(a, b, c, a * b, b * a) \in F, \\
(c * a) *(c * b) & =(c * a) *(c * T(b, a, b * a, a * b)) \\
& =T_{3}(a, b, c, a * b, b * a) \in F .
\end{aligned}
$$

Moreover, if $a$ and $c$ are comparable and $b$ and $d$ are comparable, then we apply the partial term $T_{4}$ to derive

$$
\begin{aligned}
& \min (a, c) * \min (b, d) \\
& \quad=(T(a, b, a * b, b * a) \wedge T(c, d, c * d, d * c)) *(b \wedge d)= \\
& =T_{4}(a, b, c, d, a * b, b * a, c * d, d * c) \in F .
\end{aligned}
$$

This shows $F \in$ Fil $\mathbf{P}$.

Remark 4.14 Let us consider the partial term $T(x, y, z, u):=$ $U(x, y) \wedge(z * y) \wedge u$ from the proof of Lemma 4.11 and put

$Q(x, y, z):=T(x, z, x * y, y * x)$,

i.e.,

$Q(x, y, z)=U(x, z) \wedge((x * y) * z) \wedge(y * x)$.

Of course, this is only a partial term because the infimum in $Q$ need not exists for some elements from a strongly sectionally pseudocomplemented poset $\mathbf{P}=(P, \leq, *, 1)$. It is of some interest that this partial term behaves like a Maltsev term. Namely, we can easily compute

$$
\begin{aligned}
Q(x, x, z) & =U(x, z) \wedge((x * x) * z) \wedge(x * x) \\
& =U(x, z) \wedge(1 * z) \wedge 1=U(x, z) \wedge z=z, \\
Q(x, z, z) & =U(x, z) \wedge((x * z) * z) \wedge(z * x) \\
& =(U(z, x) \wedge(z * x)) \wedge((x * z) * z)= \\
& =x \wedge((x * z) * z)=x .
\end{aligned}
$$

Moreover, these expressions $Q(x, x, z)$ and $Q(x, z, z)$ are defined for all $x, z \in P$.

For every sectionally pseudocomplemented lattice $\mathbf{L}=$ $(L, \vee, \wedge, *)$ and every $M \subseteq L$, let $F(M)$ denote the filter of L generated by $M$.

The connection between filters generated by a certain subset and congruences on sectionally pseudocomplemented lattices is described in the following proposition.

Proposition 4.15 Let $\mathbf{L}=(L, \vee, \wedge, *)$ be a sectionally pseudocomplemented lattice, $M \subseteq L$ and $a \in L$. Then,

$$
\Phi(F(M))=\Theta(M \times\{1\}),
$$

$[1](\Theta(M \times\{1\}))=F(M)$.

In particular,

$$
\Phi(F(a))=\Theta(a, 1),
$$

$[1](\Theta(a, 1))=F(a)$.

Proof Since $M \times\{1\} \subseteq \Phi(F(M))$, we have

$\Theta(M \times\{1\}) \subseteq \Phi(F(M))$

and hence

$[1](\Theta(M \times\{1\})) \subseteq[1](\Phi(F(M)))=F(M)$

according to Corollary 2.7. Because of $M \subseteq[1](\Theta(M \times$ $\{1\})$ ), we have

$F(M) \subseteq[1](\Theta(M \times\{1\}))$

and hence

$\Phi(F(M)) \subseteq \Phi([1](\Theta(M \times\{1\})))=\Theta(M \times\{1\})$

according to Corollary 2.7 .

An analogous result holds for strongly sectionally pseudocomplemented posets. 
Acknowledgements Support of the research of the authors by the Austrian Science Fund (FWF), project I 4579-N, and the Czech Science Foundation (GAČR), project 20-09869L, entitled "The many facets of orthomodularity", as well as by ÖAD, project CZ 02/2019, entitled "Function algebras and ordered structures related to logic and data fusion", and, concerning the first author, by IGA, project PřF 2021030 , is gratefully acknowledged.

Author contributions Both authors contributed equally to this manuscript.

Funding Open access funding provided by Austrian Science Fund (FWF). This study was funded by the Austrian Science Fund (FWF), project I 4579-N, and the Czech Science Foundation (GAČR), project 20-09869L, as well as by ÖAD, project CZ 02/2019, and, concerning the first author, by IGA, project PřF 2021030.

\section{Declarations}

Conflict of interest The authors declare that they have no conflict of interest.

Availability of data and material Not applicable.

Code availability: Not applicable.

Open Access This article is licensed under a Creative Commons Attribution 4.0 International License, which permits use, sharing, adaptation, distribution and reproduction in any medium or format, as long as you give appropriate credit to the original author(s) and the source, provide a link to the Creative Commons licence, and indicate if changes were made. The images or other third party material in this article are included in the article's Creative Commons licence, unless indicated otherwise in a credit line to the material. If material is not included in the article's Creative Commons licence and your intended use is not permitted by statutory regulation or exceeds the permitted use, you will need to obtain permission directly from the copyright holder. To view a copy of this licence, visit http://creativecomm ons.org/licenses/by/4.0/.

\section{References}

Birkhoff G (1979) Lattice theory. AMS Colloq. Publ. 25, Providence, R. I. ISBN 0-8218-1025-1

Chajda I (2003) An extension of relative pseudocomplementation to non-distributive lattices. Acta Sci. Math. (Szeged) 69:491-496

Chajda I, Eigenthaler G, Länger H (2012) Congruence Classes in Universal Algebra. Heldermann, Lemgo. ISBN 3-88538-226-1

Chajda I, Länger H, Paseka J (2021) Sectionally pseudocomplemented posets. Order. https://doi.org/10.1007/s11083-021-09555-6

Dilworth RP (1939) Non-commutative residuated lattices. Trans. Amer. Math. Soc. 46:426-444

Lakser H (1971) The structure of pseudocomplemented distributive lattices. I. Subdirect decomposition. Trans. Amer. Math. Soc. 156:335-342

Ursini A (1972) Sulle varietà di algebre con una buona teoria degli ideali. Boll. Un. Mat. Ital. 6:90-95

Werner H (1973) A Mal'cev condition for admissible relations. Algebra Univ. 3:263

Publisher's Note Springer Nature remains neutral with regard to jurisdictional claims in published maps and institutional affiliations. 